\title{
Trigonometric Solutions of WDVV Equations and Generalized Calogero-Moser-Sutherland Systems
}

Misha V. FEIGIN

Department of Mathematics, University of Glasgow, G12 8QW, UK

E-mail: M.Feigin@maths.gla.ac.uk

URL: http://www.maths.gla.ac.uk/ mf/

Received May 18, 2009, in final form September 07, 2009; Published online September 17, 2009 doi:10.3842/SIGMA.2009.088

\begin{abstract}
We consider trigonometric solutions of WDVV equations and derive geometric conditions when a collection of vectors with multiplicities determines such a solution. We incorporate these conditions into the notion of trigonometric Veselov system ( $\vee$-system) and we determine all trigonometric $\vee$-systems with up to five vectors. We show that generalized Calogero-Moser-Sutherland operator admits a factorized eigenfunction if and only if it corresponds to the trigonometric $\vee$-system; this inverts a one-way implication observed by Veselov for the rational solutions.
\end{abstract}

Key words: Witten-Dijkgraaf-Verlinde-Verlinde equations, V-systems, Calogero-MoserSutherland systems

2000 Mathematics Subject Classification: 35Q40; 52C99

\section{Introduction}

In this note we are interested in the special trilogarithmic solutions of the generalized WittenDijkgraaf-Verlinde-Verlinde (WDVV) equations [1]. Such solutions are determined by a finite collection $A$ of covectors $\alpha$ with multiplicities $c_{\alpha}$. More specifically, the prepotential satisfying the WDVV equations has the form

$$
F=\sum_{\alpha \in A} c_{\alpha} L i_{3}\left(e^{2 i \alpha(x)}\right)+\text { cubic terms, }
$$

where $\mathrm{Li}_{3}$ is the trilogarithm function. Solution of this type for the $A_{n}$ root system appeared in [2] in relation with Seiberg-Witten theory. More systematically such solutions were studied by Hoevenaars and Martini in [3, 4] who determined solutions for all irreducible reduced root systems [4]. More recently solutions of the form (1) were derived from reductions of Egorov hydrodynamic chains in [5].

The rational versions of solutions (1) play an important role in the theory of Frobenius manifolds, a geometric framework for the WDVV equations [6]. Thus solutions corresponding to the Coxeter root systems are almost dual to the Frobenius structures on the orbit spaces of finite Coxeter groups [7]. In the trigonometric case such a duality is verified for the affine $A_{n}$ case in $[8,9]$. The study of general rational solutions of the form

$$
F=\sum_{\alpha \in A} \alpha(x)^{2} \log \alpha(x)
$$

was initiated by Veselov in [10] where a geometric notion of the $\vee$-system equivalent to the WDVV equations for (2) was introduced. It was shown in [11] that any generalized CalogeroMoser-Sutherland (CMS) operator admitting a factorized eigenfunction determines a $\vee$-system. 
In this note we are interested in the solutions (1) where the cubic terms involve extra variable like in the works $[3,4]$ on the solutions for the root systems. We derive geometric and algebraic conditions for a system of vectors with multiplicities so that the corresponding function (1) satisfies the WDVV equations. These conditions should be thought of as trigonometric analogue of the notion of the $\mathrm{V}$-system. The conditions carry rather strong geometrical restrictions on the collection of vectors formulated in terms of series of vectors parallel to a chosen one. We illustrate this by determining all trigonometric $\vee$-systems with up to five vectors in the plane.

Trigonometric ansatz, in contrast to the rational one, allows to define the generalized CMS operator corresponding to the solution (1). We show that this operator has a factorized eigenfunction. This statement inverts the one for the rational $\vee$-systems obtained in [11]. In fact our arguments follow [11] very closely. We also discuss additional condition needed to have trigonometric solution to the WDVV equations starting from a CMS operator with factorized eigenfunction.

\section{Trigonometric $\vee$-systems}

Consider a function $F$ of the form

$$
F=\frac{1}{3} y^{3}+\sum_{\alpha \in A} c_{\alpha} \alpha(x)^{2} y+\lambda \sum_{\alpha \in A} c_{\alpha} f(\alpha(x))
$$

where $A$ is a finite collection of covectors on $V \cong \mathbb{C}^{n}, x=\left(x_{1}, \ldots, x_{n}\right), c_{\alpha}, \lambda$ are non-zero constants and function $f(x)$ satisfies $f^{\prime \prime \prime}(x)=\cot x$. The last equation fixes function $f(x)$ up to 2nd order terms which will not be important for the WDVV equations below. We may fix a choice of $f(x)$ by

$$
f(x)=\frac{1}{6} i x^{3}+\frac{1}{4} \operatorname{Li}_{3}\left(e^{-2 i x}\right) .
$$

The ansatz (3) introducing extra variable $y$ was proposed in [4] in the case of root systems $A=\mathcal{R}$. The form (3) guarantees that the matrix of third derivatives involving $y$ is constant, as we will explore below.

We will assume throughout the paper that collection $A$ of covectors $\alpha$ belongs to an $n$-dimensional lattice, and that the bilinear form

$$
(u, v):=\sum_{\alpha \in A} c_{\alpha} \alpha(u) \alpha(v)
$$

is non-degenerate on $V$. The form $(\cdot, \cdot)$ identifies $V$ and $V^{*}$ and following [10] we will denote by $\gamma^{\vee}$ the vector dual to the covector $\gamma$. We will also denote through $(\cdot, \cdot)$ the corresponding inner product on $V^{*}$. We are interested in the conditions on $\left\{\alpha, c_{\alpha}, \lambda\right\}$ when function $F$ satisfies the WDVV equations

$$
F_{i} F_{k}^{-1} F_{j}=F_{j} F_{k}^{-1} F_{i},
$$

$i, j, k=0,1, \ldots, n$. Here $F_{i}$ are $(n+1) \times(n+1)$ matrices of third derivatives in the coordinates $\left(x_{0}=y, x_{1}, \ldots, x_{n}\right),\left(F_{i}\right)_{a b}=\frac{\partial^{3} F}{\partial x_{i} \partial x_{a} \partial x_{b}}$. It is sufficient to fix $k=0$, then $F_{0}=F_{y}$ is the following non-degenerate matrix

$$
F_{y}=2\left(\begin{array}{cc}
1 & 0 \\
0 & \sum_{\alpha \in A} c_{\alpha} \alpha \otimes \alpha
\end{array}\right) .
$$


Similarly

$$
F_{i}=\left(\begin{array}{cc}
0 & 2 \sum_{\alpha \in A} c_{\alpha} \alpha_{i} \alpha \\
2 \sum_{\alpha \in A} c_{\alpha} \alpha_{i} \alpha & \lambda \sum_{\alpha \in A} c_{\alpha} \alpha_{i} \cot \alpha(x) \alpha \otimes \alpha
\end{array}\right),
$$

where we denoted by $\alpha$ both column and row vectors $\alpha=\left(\alpha_{1}, \ldots, \alpha_{n}\right)$.

The WDVV conditions for a function $F$ can be reformulated partly using geometry of the system $A$. For any $\alpha \in A$ let us collect all the covectors from $A$ non-parallel to $\alpha$ into the disjoint union of $\boldsymbol{\alpha}$-series $\Gamma_{\alpha}^{1}, \ldots, \Gamma_{\alpha}^{k}$. These series are determined by the property that for any $s=1, \ldots, k$ and for any two covectors $\gamma_{1}, \gamma_{2} \in \Gamma_{\alpha}^{s}$ one has either $\gamma_{1}-\gamma_{2}=n \alpha$ or $\gamma_{1}+\gamma_{2}=n \alpha$ for some integer $n$. We also assume that the series are maximal, that is if $\beta \in \Gamma_{\alpha}^{i}$ then $\Gamma_{\alpha}^{i}$ must contain all the vectors of the form $\pm \beta+n \alpha \in A$ with $n \in \mathbb{Z}$.

We note that solution (3) is not affected if some of the covectors $\alpha \in A$ are replaced with $-\alpha$. By appropriate choice of signs the vectors can be made to belong to a half-space, we will denote such systems as $A_{+}$. Moreover, for any $\alpha \in A$ one can choose a positive system $A_{+} \ni \alpha$ in such a way that $\alpha$-series $\Gamma_{\alpha}^{s}$ will consist of vectors of the form $\beta_{s}+n_{i} \alpha \in A_{+}$for appropriate integer parameters $n_{i}$ with $\beta_{s} \in A_{+}$.

Definition 1. Let $A \subset V^{*} \cong \mathbb{C}^{n}$ be a finite collection of covectors $\alpha$ with multiplicities $c_{\alpha}$ such that the corresponding form (4) is non-degenerate and the covectors $\alpha$ belong to an $n$ dimensional lattice. We say that $A$ is a trigonometric $\vee$-system if for any $\alpha \in A$ and for any $\alpha$-series $\Gamma_{\alpha}^{s}$ one has

$$
\sum_{\beta \in \Gamma_{\alpha}^{s}} c_{\beta}(\alpha, \beta) \alpha \wedge \beta=0 .
$$

Notice that $\alpha \wedge \beta_{1}= \pm \alpha \wedge \beta_{2}$ if $\beta_{1}, \beta_{2}$ belong to the same $\alpha$-series $\Gamma_{\alpha}^{s}$ so identities (6) may be simplified accordingly. Also replacement of some of the covectors by their opposite preserves the class of trigonometric $\vee$-systems. Note also that the non-degenerate linear transformations act naturally on the trigonometric $\vee$-systems, and that the direct sum $A_{1} \oplus A_{2}$ of the trigonometric $\vee$-systems $A_{1} \subset V_{1}^{*}, A_{2} \subset V_{2}^{*}$ considered as a set of covectors in $V_{1} \oplus V_{2}$ is again a trigonometric $\checkmark$-system. The systems obtained in this way will be called reducible. If such a decomposition is not possible then the (trigonometric $\vee$-)system is called irreducible.

Theorem 1. The WDVV equations

$$
F_{i} F_{y}^{-1} F_{j}=F_{j} F_{y}^{-1} F_{i},
$$

$i, j=0,1, \ldots, n$, for the function (3) are equivalent to the following two conditions:

1) $A$ is a trigonometric $\vee$-system;

2) for a positive system $A_{+}$and for any vectors $a, b, c, d \in V$

$$
\sum_{\alpha, \beta \in A_{+}}\left(\frac{1}{4} \lambda^{2}(\alpha, \beta)-1\right) c_{\alpha} c_{\beta} B_{\alpha, \beta}(a, b) B_{\alpha, \beta}(c, d)=0,
$$

where $B_{\alpha, \beta}(a, b)=\alpha \wedge \beta(a, b)=\alpha(a) \beta(b)-\alpha(b) \beta(a)$.

Proof. For a vector $a \in V$ we define $F_{a}^{\vee}=F_{y}^{-1} F_{a}$ where $F_{a}=\sum_{i=1}^{n} a_{i} F_{i}$. The WDVV equations are equivalent to the commutativity $\left[F_{a}^{\vee}, F_{b}^{\vee}\right]=0$ for any $a, b \in V$. We have

$$
F_{i}^{\vee}=\left(\begin{array}{cc}
0 & \sum_{\alpha \in A} c_{\alpha} \alpha_{i} \alpha \\
\sum_{\alpha \in A} c_{\alpha} \alpha_{i} \alpha^{\vee} & \frac{\lambda}{2} \sum_{\alpha \in A} c_{\alpha} \alpha_{i} \cot \alpha(x) \alpha \otimes \alpha^{\vee}
\end{array}\right),
$$


where $\alpha^{\vee}$ is the (column) vector dual to the (row) covector $\alpha$ under the bilinear form $G=$ $\sum_{\alpha \in A} c_{\alpha} \alpha \otimes \alpha$. Therefore

$$
F_{a}^{\vee}=\left(\begin{array}{cc}
0 & \sum_{\alpha \in A} c_{\alpha} \alpha(a) \alpha \\
\sum_{\alpha \in A} c_{\alpha} \alpha(a) \alpha^{\vee} & \frac{\lambda}{2} \sum_{\alpha \in A} c_{\alpha} \alpha(a) \cot \alpha(x) \alpha \otimes \alpha^{\vee}
\end{array}\right)
$$

for any $a \in \mathbb{C}^{n}$. Now the product $F_{a}^{\vee} F_{b}^{\vee}$ equals

$$
\left(\begin{array}{cc}
\sum_{\alpha, \beta \in A} c_{\alpha} c_{\beta} \alpha(a) \beta(b) \alpha\left(\beta^{\vee}\right) & \frac{\lambda}{2} \sum_{\alpha, \beta \in A} c_{\alpha} c_{\beta} \alpha(a) \beta(b) \alpha\left(\beta^{\vee}\right) \cot \beta(x) \beta \\
\frac{\lambda}{2} \sum_{\alpha, \beta \in A} c_{\beta} c_{\alpha} \alpha(a) \beta(b) \alpha\left(\beta^{\vee}\right) \cot \alpha(x) \alpha^{\vee} & \sum_{\alpha, \beta \in A} c_{\alpha} c_{\beta} \alpha(a) \beta(b) \alpha^{\vee} \otimes \beta \\
+\frac{\lambda^{2}}{4} & \sum_{\alpha, \beta \in A} c_{\alpha} c_{\beta} \alpha(a) \beta(b) \alpha\left(\beta^{\vee}\right) \cot \alpha(x) \cot \beta(x) \beta \otimes \alpha^{\vee}
\end{array}\right) .
$$

Therefore $\left[F_{a}^{\vee}, F_{b}^{\vee}\right]=0$ is equivalent to the identities

$$
\begin{aligned}
& \sum_{\alpha, \beta \in A} c_{\alpha} c_{\beta} B_{\alpha, \beta}(a, b)(\alpha, \beta) \cot \alpha(x) \alpha^{\vee}=0, \\
& \sum_{\alpha, \beta \in A}\left(\frac{\lambda^{2}}{4} c_{\alpha} c_{\beta}(\alpha, \beta) \cot \alpha(x) \cot \beta(x)+c_{\alpha} c_{\beta}\right) B_{\alpha, \beta}(a, b) \alpha \wedge \beta=0 .
\end{aligned}
$$

To cancel singularities in (9) one should have

$$
\sum_{\substack{\beta \in A \\ \beta \nsim \alpha}} c_{\beta}(\alpha, \beta) \cot \beta(x) B_{\alpha, \beta}(a, b) \alpha \wedge \beta=0
$$

when $\cot \alpha(x)=0$. A linear combination of functions $\left.\cot \beta(x)\right|_{\cot \alpha(x)=0}$ can vanish only if it vanishes for each $\alpha$-series:

$$
\sum_{\beta \in \Gamma_{\alpha}^{s}} c_{\beta}(\alpha, \beta) \cot \beta(x) B_{\alpha, \beta}(a, b) \alpha \wedge \beta=0
$$

for all $\alpha$-series $\Gamma_{\alpha}^{s}$ (see e.g. [14] for more detailed explanation). The last relation can be simplified as

$$
\sum_{\beta \in \Gamma_{\alpha}^{s}} c_{\beta}(\alpha, \beta) \alpha \wedge \beta=0,
$$

which means that $A$ is a trigonometric $\vee$-system. Identities (10) guarantee that the left-hand side of (9) is non-singular. Since all the vectors from $A$ belong to an $n$-dimensional lattice with basis $e^{1}, \ldots, e^{n}$, the left-hand side of (9) is a rational function in the exponential coordinates $e^{e^{i}(x)}$. This rational function has degree zero and therefore it is a constant. We can assume that all covectors from $A$ belong to a half-space hence form a positive system $A_{+}$, so in appropriate limit $\cot (\alpha, x) \rightarrow i$ for all $\alpha \in A_{+}$. Thus property (9) is equivalent to (10) together with the condition

$$
\sum_{\alpha, \beta \in A_{+}}\left(\frac{\lambda^{2}}{4} c_{\alpha} c_{\beta}(\alpha, \beta)-c_{\alpha} c_{\beta}\right) B_{\alpha, \beta}(a, b) \alpha \wedge \beta=0 .
$$

The remaining condition (8) is equivalent to the set of properties

$$
\sum_{\beta \in A} c_{\beta}(\alpha, \beta) B_{\alpha, \beta}(a, b)=0,
$$

for any $\alpha \in A$. Identities (11) follow from the $\vee$-conditions (10), this completes the proof of the theorem. 
Remark 1. Let trigonometric $\vee$-systems $A_{1} \subset V_{1}^{*}, A_{2} \subset V_{2}^{*}$ define the solutions (3) of the WDVV equations for some $\lambda_{1}, \lambda_{2}$. Then the trigonometric $\vee$-system $A_{1} \oplus A_{2}$ does not define a solution. Indeed, let us take vectors $a, c \in V_{1}$ and $b, d \in V_{2}$. Then property (7) implies that

$$
(a, c)_{1}(b, d)_{2}=0
$$

where $(\cdot, \cdot)_{1,2}$ are $\vee$-forms (4) in the corresponding spaces $V_{1,2}$. Clearly, the relation (12) does not hold for general vectors $a, b, c, d$.

Remark 2. Not all the trigonometric solutions of the WDVV equations have the form (3). It is shown in [15] that trilogarithmic functions have to arise when ansatz for $F$ is given by summation of $g((\alpha, x))$ over the roots of a root system, $x \in V$.

Remark 3. A slightly more general ansatz for the solutions $F$ can be considered when cubic terms in $x$ are added to $F$. Similarly to the proof of Theorem 1 it follows that $A$ still has to be a trigonometric $\vee$-system. The almost dual potentials corresponding to the $A_{n}$ affine Weyl group orbit spaces have such a form [9]. The corresponding trigonometric $\vee$-system $A$ is the $A_{n}$ root system in this case.

Proposition 1. Let $A=\left\{\alpha, c_{\alpha}\right\}$ be a trigonometric $\vee$-system. Then the set of vectors $\left\{\sqrt{c_{\alpha}} \alpha\right\}$ is a (rational) $\vee$-system, that is $F^{\mathrm{rat}}=\sum_{\alpha \in A} c_{\alpha} \alpha(x)^{2} \log \alpha(x)$ is a solution of the WDVV equations in the space $V$.

Proof. By definition of the trigonometric $\vee$-system for any $\alpha \in A$ relations (6) hold. Consider two-dimensional plane $\pi \subset V$ and sum up relations (6) over $s$ so that the $\alpha$-series $\Gamma_{\alpha}^{s}$ belong to the plane $\pi \ni \alpha$. We arrive at the relations

$$
\sum_{\beta \in A \cap \pi} c_{\beta}(\alpha, \beta) \alpha \wedge \beta=0
$$

or, equivalently,

$$
\sum_{\beta \in A \cap \pi} c_{\beta}(\alpha, \beta) \beta \quad \text { is proportional to } \alpha .
$$

Relations (13) is a definition of the (rational) $\vee$-system for the set of covectors $\left\{\sqrt{c_{\alpha}} \alpha\right\}$ (see [10] and [13] for the complex space). It is equivalent to the property that $F^{\text {rat }}$ satisfies WDVV equations in the space $V[10,13]$. Proposition is proven.

Due to existence of extra variable $y$ in the ansatz (3) the WDVV equations are nontrivial already when $n=2$. Thus it is natural to study at first two-dimensional configurations $A$ defining solutions of WDVV equations. When $A$ consists of one vector the corresponding form (4) is degenerate. If $A$ consists of two non-collinear vectors $\alpha, \beta$ then it follows that $(\alpha, \beta)=0$ therefore relation (6) holds and $A$ is a trigonometric $\vee$-system. However relation (7) cannot hold then for any $\lambda$ and therefore a pair of vectors does not define a solution to WDVV equations (see also Remark 1 above). The following propositions deal with the next simplest cases when $A$ consists of 3,4 and 5 vectors respectively. In fact all irreducible trigonometric $\vee$-systems with up to 5 covectors have to be two-dimensional.

Proposition 2. Let system $A$ consist of three vectors $\alpha, \beta, \gamma$ with nonzero multiplicities $c_{\alpha}$, $c_{\beta}, c_{\gamma}$. Then $A$ is an irreducible trigonometric $\vee$-system iff $\alpha \pm \beta \pm \gamma=0$ for some choice of signs. The non-degeneracy condition for the form (4) is then given by $c_{\alpha} c_{\beta}+c_{\alpha} c_{\gamma}+c_{\beta} c_{\gamma} \neq 0$.

Any such system $A$ defines the solution (3) of the WDVV equations with $\lambda=2\left(c_{\alpha} c_{\beta}+c_{\alpha} c_{\gamma}+\right.$ $\left.c_{\beta} c_{\gamma}\right)\left(c_{\alpha} c_{\beta} c_{\gamma}\right)^{-1 / 2}$. 
Proof. It follows from relations (6) that $\gamma=\alpha+\beta$ up to multiplication of some of the vectors by -1 . We take a basis $e^{1}=\alpha, e^{2}=\beta$ in $\mathbb{C}^{2}$. The bilinear form (4) takes the form $G=$ $c_{\alpha} x_{1}^{2}+c_{\beta} x_{2}^{2}+c_{\gamma}\left(x_{1}+x_{2}\right)^{2}$. This form is non-degenerate iff $c_{\alpha} c_{\beta}+c_{\alpha} c_{\gamma}+c_{\beta} c_{\gamma} \neq 0$. One can check that

$$
e^{1^{\vee}}=\frac{\left(c_{\beta}+c_{\gamma}\right) e_{1}-c_{\gamma} e_{2}}{c_{\alpha} c_{\beta}+c_{\alpha} c_{\gamma}+c_{\beta} c_{\gamma}}, \quad e^{2 \vee}=\frac{-c_{\gamma} e_{1}+\left(c_{\alpha}+c_{\gamma}\right) e_{2}}{c_{\alpha} c_{\beta}+c_{\alpha} c_{\gamma}+c_{\beta} c_{\gamma}},
$$

where $e_{1}, e_{2}$ is dual basis to $e^{1}, e^{2}$, that is $e^{i}\left(e_{j}\right)=\delta_{j}^{i}$. Relations (6) look as follows

$$
\begin{aligned}
& \left(e^{1 \vee}, e^{2^{\vee}}\right)\left(c_{\beta}+c_{\gamma}\right)+\left(e^{1 \vee}, e^{1 \vee}\right) c_{\gamma}=0, \\
& \left(e^{1 \vee}, e^{2 \vee}\right)\left(c_{\alpha}+c_{\gamma}\right)+\left(e^{2 \vee}, e^{2 \vee}\right) c_{\gamma}=0, \\
& \left(e^{1 \vee}, e^{2 \vee}\right)\left(c_{\alpha}-c_{\beta}\right)+\left(e^{1 \vee}, e^{1 \vee}\right) c_{\alpha}-\left(e^{2 \vee}, e^{2^{\vee}}\right) c_{\beta}=0,
\end{aligned}
$$

and they are automatically satisfied. Relation (7) results to one scalar equation

$$
\frac{\lambda^{2}}{4}\left(c_{\alpha} c_{\beta}\left(\alpha^{\vee}, \beta^{\vee}\right)+c_{\alpha} c_{\gamma}\left(\alpha^{\vee}, \gamma^{\vee}\right)+c_{\beta} c_{\gamma}\left(\beta^{\vee}, \gamma^{\vee}\right)\right)=c_{\alpha} c_{\beta}+c_{\alpha} c_{\gamma}+c_{\beta} c_{\gamma},
$$

which has solution as stated in the formulation.

In the following proposition we study configurations consisting of four covectors.

Proposition 3. Let system $A$ consist of four vectors $\alpha, \beta, \gamma, \delta$ with nonzero multiplicities $c_{\alpha}$, $c_{\beta}, c_{\gamma}, c_{\delta}$. Then $A$ is an irreducible trigonometric $\vee$-system iff the vectors in $A_{+}$take the form $e^{1}$, $e^{2}, e^{1} \pm e^{2}$ in a suitable basis, and the corresponding multiplicities $c_{1}, c_{2}, c_{ \pm}$satisfy $c_{1}=c_{2}$. This property is equivalent to the orthogonality $\left(e^{1}+e^{2}, e^{1}-e^{2}\right)=0$ under the corresponding $\vee$-product. The non-degeneracy condition for the form (4) is then given by $\Delta=\left(c_{1}+2 c_{+}\right)\left(c_{1}+2 c_{-}\right) \neq 0$.

These systems $A$ define the solutions (3) of the WDVV equations with $\lambda=2 \Delta c_{1}^{-1 / 2}\left(4 c_{+} c_{-}+\right.$ $\left.c_{1}\left(c_{+}+c_{-}\right)\right)^{-1 / 2}$ once $\lambda$ is finite.

Proof. It follows from the series relations (6) that there is a vector $\alpha \in A$ such that all the remaining vectors $\beta, \gamma, \delta \in A$ belong to single $\alpha$-series $\Gamma_{\alpha}^{1}$.

Indeed, otherwise, up to renaming the covectors and taking opposite, we have $\delta=\gamma+n \alpha$, $n \in \mathbb{N},(\alpha, \beta)=(\gamma, \delta)=0$. Then consideration of $\beta$-series gives $2 \gamma+n \alpha=m \beta$ for some $m \in \mathbb{Z}$. And consideration of $\gamma$-series gives $\alpha+p \gamma= \pm \beta$ for some $p \in \mathbb{Z}$. Therefore $2 \gamma+n \alpha= \pm m(\alpha+p \gamma)$, hence $n= \pm m$ and $2= \pm m p$, thus $m= \pm 1$ or $p= \pm 1$. In the case $m= \pm 1$ we have $n=1$ hence $\gamma$-series contains $\delta$ together with $\alpha$ and $\beta$. And in the case $p= \pm 1$ the $\alpha$-series contains $\beta$ together with $\gamma$ and $\delta$.

So we can assume that there is only one $\alpha$-series so that the remaining vectors take the form $\gamma=\beta+n_{1} \alpha, \delta=\beta+n_{2} \alpha$ with integer $n_{2}>n_{1}>0$. By considering $\beta$-series we conclude that $n_{1}=1$. Consider now the $\delta$-series. It is easy to see that covector $\beta$ has to form a single series, therefore $(\beta, \delta)=0$ and the covectors $\beta+\alpha$ and $\alpha$ belong to a $\delta$-series. This is possible only if $n_{2}=2$. Taking now the basis vectors as $e^{1}=\alpha, e^{2}=\beta+\alpha$ we conclude that the system $A$ consists of covectors $e^{1}, e^{2}, e^{1} \pm e^{2}$.

The bilinear form (4) takes now the form

$$
\begin{aligned}
G & =c_{1} x_{1}^{2}+c_{2} x_{2}^{2}+c_{+}\left(x_{1}+x_{2}\right)^{2}+c_{-}\left(x_{1}-x_{2}\right)^{2} \\
& =\left(c_{1}+c_{+}+c_{-}\right) x_{1}^{2}+\left(c_{2}+c_{+}+c_{-}\right) x_{2}^{2}+2\left(c_{+}-c_{-}\right) x_{1} x_{2},
\end{aligned}
$$

which has determinant $\Delta=c_{1} c_{2}+\left(c_{1}+c_{2}\right)\left(c_{+}+c_{-}\right)+4 c_{+} c_{-}$. Therefore

$$
\begin{array}{ll}
\left(e^{1}, e^{1}\right) & =\Delta^{-1}\left(c_{2}+c_{+}+c_{-}\right), \quad\left(e^{2}, e^{2}\right)=\Delta^{-1}\left(c_{1}+c_{+}+c_{-}\right), \\
\left(e^{1}, e^{2}\right) & =\Delta^{-1}\left(c_{-}-c_{+}\right) .
\end{array}
$$


Now we analyze the series relations (6). The orthogonality $\left(e^{1}-e^{2}, e^{1}+e^{2}\right)=0$ is clearly equivalent to the condition $c_{1}=c_{2}$. Then the remaining conditions (6) on $\left(e^{1} \pm e^{2}\right)$-series are automatically satisfied. The condition (6) for the $e^{1}$-series has the form

$$
c_{-}\left(-e^{1}+e^{2}, e^{1}\right)+c_{2}\left(e^{2}, e^{1}\right)+c_{+}\left(e^{1}+e^{2}, e^{1}\right)=0,
$$

and it follows from the scalar products (14). The condition on the $e^{2}$-series is also satisfied.

It is easy to check that relation (7) holds iff $\lambda$ is as stated, hence proposition is proven.

Proposition 4. Let irreducible trigonometric $\vee$-system A consist of five vectors with non-zero multiplicities. Then in the appropriate basis $A_{+}$takes the form $e^{1}, 2 e^{1}, e^{2}, e^{1} \pm e^{2}$ and the corresponding multiplicities $c_{1}, \widetilde{c}_{1}, c_{2}, c_{ \pm}$satisfy $c_{+}=c_{-}$(equivalently, $\left(e^{1}, e^{2}\right)=0$ ) and $2 \widetilde{c}_{1} c_{2}=$ $c_{+}\left(c_{1}-c_{2}\right)$.

The form (4) is then non-degenerate when $\Delta=\left(c_{1}+4 \widetilde{c}_{1}+2 c_{+}\right)\left(c_{2}+2 c_{+}\right) \neq 0$. The corresponding solution of the WDVV equations has the form (3) with $\lambda=\sqrt{2} \Delta\left(c_{2}+2 c_{+}\right)^{-1 / 2}\left(c_{1}+\right.$ $\left.4 \widetilde{c}_{1}\right)^{-1 / 2} c_{+}^{-1 / 2}$.

Proof is obtained by simple analysis of the series conditions (6). One can firstly establish that $A$ is two-dimensional. Then it is easy to see that $A$ has to contain collinear vectors, and the required form follows.

To conclude this section we present a few examples of trigonometric $\vee$-systems on the plane with higher number of vectors. Recall firstly that the positive roots of the root system $\mathcal{G}_{2}$ can be written as $\alpha, \beta, \beta+\alpha, \beta+n \alpha, \beta+(n+1) \alpha, 2 \beta+(n+1) \alpha$ where $n=2$. One can show that for integer $n>2$ the above vectors never form a trigonometric $\vee$-system, and that for $n=2$ the multiplicities have to satisfy $c_{\alpha}=c_{\beta+\alpha}=c_{\beta+n \alpha}$ and $c_{\beta}=c_{2 \beta+(n+1) \alpha}=c_{\beta+(n+1) \alpha}$ which is the case of the $\mathcal{G}_{2}$ system.

There are though some possibilities to extend the $\mathcal{G}_{2}$ system. Firstly, one can show that $\mathcal{G}_{2} \cup \mathcal{A}_{2}$ where the system $\mathcal{A}_{2}$ consists of doubled short roots of $\mathcal{G}_{2}$, is a trigonometric $\vee$-system for appropriate multiplicities. Secondly the following proposition takes place.

Proposition 5. Let $A$ consist of the vectors $e_{1}, e_{2}, 2 e_{2}, \frac{1}{2}\left(e_{1} \pm e_{2}\right), \frac{1}{2}\left(e_{1} \pm 3 e_{2}\right)$ with the corresponding nonzero multiplicities $c_{1}, c_{2}, \widetilde{c}_{2}, a, b$. Then $A$ is a trigonometric $\vee$-system iff the multiplicities satisfy the relations $a=3 b, c_{2}=a+2 \widetilde{c}_{2},\left(2 c_{1}+b\right) c_{2}=\left(c_{1}+2 b\right) a$.

Note that in the limiting case $\widetilde{c}_{2}=0$ we recover the system $\mathcal{G}_{2}$ with special multiplicities.

An example of trigonometric $\checkmark$-system with yet higher number of vectors is given by vectors $e_{1}, 2 e_{1}, e_{2}, 2 e_{2}, e_{1} \pm e_{2}, e_{1} \pm 2 e_{2}, 2 e_{1} \pm e_{2}$ where the multiplicities can be chosen appropriately.

\section{Relations with generalized Calogero-Moser-Sutherland systems}

Relation between $\vee$-systems and the property of a Schrödinger operator of CMS type to have a factorized eigenfunction was observed by Veselov in [11]. Namely, it was shown in [11] that if an operator

$$
L=-\Delta+\sum_{\alpha \in A_{+}} \frac{m_{\alpha}\left(m_{\alpha}+1\right)(\alpha, \alpha)}{\sin ^{2}(\alpha, x)}
$$

has a formal eigenfunction

$$
\psi=\prod_{\alpha \in A_{+}} \sin ^{-m_{\alpha}}(\alpha, x), \quad L \psi=\mu \psi,
$$


then $F=\sum_{\alpha \in A_{+}} m_{\alpha}(\alpha, x)^{2} \log (\alpha, x)$ satisfies the WDVV equations. The following theorem establishes the converse statement in the case of trigonometric $\vee$-systems.

Theorem 2. Let $A$ be a trigonometric $\vee$-system consisting of pairwise non-collinear covectors $\alpha$ with multiplicities $c_{\alpha}$. Then Schrödinger operator

$$
L=-\Delta+\sum_{\alpha \in A} \frac{c_{\alpha}\left(c_{\alpha}+1\right)(\alpha, \alpha)}{\sin ^{2} \alpha(x)}
$$

constructed by the metric (4) has the formal eigenfunction

$$
\psi=\prod_{\alpha \in A} \sin ^{-c_{\alpha}} \alpha(x), \quad L \psi=\mu \psi .
$$

Proof. The property $L \psi=\mu \psi$ is equivalent to the identity

$$
\sum_{\alpha \neq \beta} c_{\alpha} c_{\beta}(\alpha, \beta) \cot \alpha(x) \cot \beta(x)=\text { const. }
$$

To establish the last identity it is sufficient to show that the left-hand side of (17) is non-singular. In other words, we need to show that

$$
\sum_{\beta, \beta \neq \alpha} c_{\beta}(\alpha, \beta) \cot \beta(x)=0
$$

if $\cot \alpha(x)=0$. The last properties are sufficient to check when summation is taken along arbitrary $\alpha$-series, then it is guaranteed by relation (6). This proves the theorem.

Corollary 1. Assume that function (3) constructed by a set of pairwise non-collinear covectors $\alpha$ with multiplicities $c_{\alpha}$ satisfies the WDVV equations (5). Then relation (16) holds for the Schrödinger operator (15).

Conversely, the property of a Schrödinger operator to have a factorized eigenfunction implies that the corresponding vectors $\sqrt{c_{\alpha}} \alpha$ form a rational $\vee$-system [11]. This property is also sufficient to obtain the trigonometric $\vee$-system, and the arguments are close to [11].

Theorem 3. Assume that the Schrödinger operator (15) has an eigenfunction (16). Then the set $A$ of vectors $\alpha$ with the multiplicities $c_{\alpha}$ forms the trigonometric $\vee$-system.

Proof. From equation (16), (15) it follows identity (18) at $\cot \alpha(x)=0$. Therefore for each $\alpha$-series $\Gamma_{\alpha}^{s}$ we have

$$
\sum_{\beta \in \Gamma_{\alpha}^{s}} c_{\beta}(\alpha, \beta) \alpha \wedge \beta=0 .
$$

Let $\beta^{\mathrm{w}}$ denote a vector dual to $\beta$ with respect to the inner product $(\cdot, \cdot)$ involved in the Schrödinger equation. By summing identities (19) along all the $\alpha$-series we conclude that

$$
\sum_{\beta \in A} c_{\beta} \beta\left(\alpha^{\mathrm{w}}\right) \beta^{\mathrm{w}} \quad \text { is proportional to } \alpha^{\mathrm{w}} \text {. }
$$

Now we can decompose the space $V=V_{1} \oplus \cdots \oplus V_{k}$ so that the operator $\sum_{\beta \in A} c_{\beta} \beta \otimes \beta^{\text {w }}$ is equal to constant $\mu_{i}$ on $V_{i}$. We can also assume that $\left(V_{i}, V_{j}\right)=0$ if $i \neq j$. It follows from (20) that $\left.G(\cdot, \cdot)\right|_{V_{i}}=\left.\mu_{i}(\cdot, \cdot)\right|_{V_{i}}$. Therefore identities (19) imply

$$
\sum_{\beta \in \Gamma_{\alpha}^{s}} c_{\beta} \alpha\left(\beta^{\vee}\right) \alpha \wedge \beta=0
$$

which are identities (6) from the definition of the trigonometric $\vee$-systems. 
Corollary 2. Assume that the Schrödinger operator (15) has an eigenfunction (16). Assume also that the system $A$ is irreducible and that for some $\Lambda$ and any $a, b, c, d \in V$ the property

$$
\sum_{\alpha, \beta \in A_{+}}(\Lambda(\alpha, \beta)-1) c_{\alpha} c_{\beta} B_{\alpha, \beta}(a, b) B_{\alpha, \beta}(c, d)=0
$$

holds. Then the corresponding function (3) with appropriate $\lambda$ satisfies the WDVV equations (5).

Remark 4. The previous corollary also holds for the reducible systems $A$ if we replace the Schrödinger equation metric $(\alpha, \beta)$ in $(21)$ by the $\vee$-product $\alpha\left(\beta^{\vee}\right)$. In this case $\lambda=2 \sqrt{\Lambda}$.

\section{Concluding remarks}

Trigonometric $\vee$-systems require further investigations. It would be interesting to obtain almost dual prepotentials for the Frobenius manifolds of the affine Weyl groups as well as for their discriminants (cf. rational case [7, 12]). Comparison with a recent work on the elliptic solutions [16] might also be interesting. We also hope that the series conditions would allow understanding and eventually classification of the trigonometric $\vee$-systems. We hope to return to some of these questions soon.

\section{Acknowledgements}

I am very grateful to L. Hoevenaars, A. Kirpichnikova, M. Pavlov, I. Strachan and A.P. Veselov for useful and stimulating discussions. The work was partially supported by the EPSRC grant EP/F032889/1, by European research network ENIGMA (contract MRTN-CT-2004-5652), by PMI2 Project funded by the UK Department for Innovation, Universities and Skills for the benefit of the Japanese Higher Education Sector and the UK Higher Education Sector.

\section{References}

[1] Marshakov A., Mironov A., Morozov A., WDVV-like equations in $N=2$ SUSY Yang-Mills theory, Phys. Lett. B 389 (1996), 43-52, hep-th/9607109.

[2] Marshakov A., Mironov A., Morozov A., More evidence for the WDVV equations in $N=2$ SUSY Yang-Mills theories, Internat. J. Modern Phys. A 15 (2000), 1157-1206, hep-th/9701123.

[3] Hoevenaars L.K., Martini R., On the WDVV equations in five-dimensional gauge theories, Phys. Lett. B 557 (2003), 94-104, math-ph/0212016.

[4] Martini R., Hoevenaars L.K., Trigonometric solutions of the WDVV equations from root systems, Lett. Math. Phys. 65 (2003), 15-18, math-ph/0302059.

[5] Pavlov M., Explicit solutions of the WDVV equation determined by the "flat" hydrodynamic reductions of the Egorov hydrodynamic chains, nlin.SI/0606008.

[6] Dubrovin B., Geometry of 2D topological field theories, in Integrable Systems and Quantum Groups (Montecatini Terme, 1993), Lecture Notes in Math., Vol. 1620, Springer, Berlin, 1996, 120-348, hep-th/9407018.

[7] Dubrovin B., On almost duality for Frobenius manifolds, in Geometry, Topology, and Mathematical Physics, Amer. Math. Soc. Transl. Ser. 2, Vol. 212, Amer. Math. Soc., Providence, RI, 2004, 75-132, math.DG/0307374.

[8] Riley A., Frobenius manifolds: caustic submanifolds and discriminant almost duality, Ph.D. Thesis, Hull University, 2007.

[9] Riley A., Strachan I.A.B., A note on the relationship between rational and trigonometric solutions of the WDVV equations, J. Nonlinear Math. Phys. 14 (2007), 82-94, nlin.SI/0605005.

[10] Veselov A.P., Deformations of the root systems and new solutions to generalised WDVV equations, Phys. Lett. A 261 (1999), 297-302, hep-th/9902142. 
[11] Veselov A.P., On generalizations of the Calogero-Moser-Sutherland quantum problem and WDVV equations, J. Math. Phys. 43 (2002), 5675-5682, math-ph/0204050.

[12] Feigin M.V., Veselov A.P., Logarithmic Frobenius structures and Coxeter discriminants, Adv. Math. 212 (2007), 143-162, math-ph/0512095.

[13] Feigin M.V., Veselov A.P., On the geometry of V-systems, in Geometry, Topology, and Mathematical Physics, Amer. Math. Soc. Transl. Ser. 2, Vol. 224, Amer. Math. Soc., Providence, RI, 2008, 111-123, arXiv:0710.5729.

[14] Feigin M.V., Bispectrality for deformed Calogero-Moser-Sutherland systems, J. Nonlinear Math. Phys. 12 (2005), suppl. 2, 95-136, math-ph/0503020.

[15] Braden H., Marshakov A., Mironov A., Morozov A., Seiberg-Witten theory for a non-trivial compactification from five to four dimensions, Phys. Lett. B 448 (1999), 195-202, hep-th/9812078.

[16] Strachan I.A.B., Weyl groups and elliptic solutions of the WDVV equations, arXiv:0802.0388. 